\title{
Open Source BioImage Informatics: Tools for Interoperability
}

\author{
Curtis T. Rueden, Johannes Schindelin, Barry E. Dezonia, Aivar R. Grislis, Mark C. Hiner, and
} Kevin W Eliceiri

Laboratory for Optical and Computational Instrumentation, University of Wisconsin at Madison, Madison, Wisconsin, USA

Biological imaging has greatly advanced over the last thirty years with the now unprecedented ability to track biological phenomena in high resolution in physiologically relevant conditions over time and in space. As these imaging technologies mature and become main stream tools for the bench biologist there is great need for improved software tools that drive the informatics workflow of the imaging process from acquisition and image analysis to visualization and dissemination. To best meet the workflow challenges, these tools need to be freely available, open source, and transparent in their development and deployment [1]. In particular it is clear that given the complexity, and heterogeneity of the modern image dataset, there cannot be a single software solution. Different imaging processing and visualization approaches need access not only to the data but also to each other. There needs to be compatibility not only in file import and export but interoperability in preserving and communicating what was done to the image. There is a great opportunity in achieving this interoperability, tools that can talk to each other not only enable new biological discovery but also efficiencies in sharing code and in many cases more precise workflows. We present our efforts towards interoperability in the Fiji ImageJ [2] and Open Microscopy Environment [3] consortiums. The consortiums are actively developing key software libraries like ImgLib [4] and Bio-Formats [5] that are utilized in dozens of software applications to parse and visualize biological image data [6], to the developmental benefit of not only of the applications but the libraries themselves.

We are developing a complete, open source system for handling biomedical images, including image acquisition, data storage, metadata (experimental data associated with an image), visualization, analysis, annotation, and database interconnectivity. We are adding support for multidimensional file structures such as fluorescence lifetime imaging and improving performance for handling large files. We are developing ImageJ2 [7] as the next generation version of ImageJ with an emphasis on performance for large multidimensional files and interoperability. Image $\mathrm{J} 2$ is a new version of Image J seeking to strengthen both the software and its community. Internally, it is a total redesign of ImageJ, but it is backwards compatible with Image J 1.x via a "legacy layer" and features a user interface closely modeled after the original (Figure 1). Under the hood, ImageJ2 completely isolates the image processing logic from the graphical user interface (UI), allowing ImageJ2 plugins to be used in many contexts, including headless in the cloud or on a server such as OMERO [8], or from within another application such as CellProfiler [9]. ImageJ2 has an N-dimensional data model driven by the powerful ImgLib2 library, which supports image data expressed in an extensible set of numeric and non-numeric types, and accessed from an extensible set of data sources. Image 2 is driven by a state-of-the-art, collaborative development process, including version control, unit testing, automated builds via a continuous integration system, a bug tracker and more. We are collaborating closely with related projects including Fiji, Bio-Formats and OMERO, and are striving to deliver a coherent software stack reusable throughout 
the life sciences community and beyond. The result is well-designed, community-driven software accessible to users yet powerful enough for programmers. We acknowledge our grant support [10].

eferences:

[1] AE Carpenter, L Kamentsky, KW Eliceiri, Nat Methods, 9 (2012), p. 666-70.

[2] J Schindelin, I Arganda-Carrera, E Frise, et al, Nat Methods, 9 (2012), p. 676-82.

[3] JR Swedlow, KW Eliceiri, Trends Cell Biol, 11 (2009), p. 656-60.

[4] T Pietzsch, S Preibisch, P Tomancak, Bioinformatics, 22 (2012), p. 3009-11.

[5] M Linkert, CT Rueden, C Allan, et al, J Cell Biol, 5 (2010), p. 777-82.

[6] KW Eliceiri, MR Berthold, IG Goldberg, et al, Nat Methods, 9 (2012), p. 697-710.

[7] http://developer.imagej.net/

[8] C Allan, JM Burel, J Moore, et al, Nat Methods, 9 (2012), p. 245-53.

[9] L Kamentsky, TR Jones, A Fraser, et al, Bioinformatics, 27 (2011), p. 1179-80.

[10] The authors acknowledge funding from NIH grants R01-CA136590 and RC2GM092519.

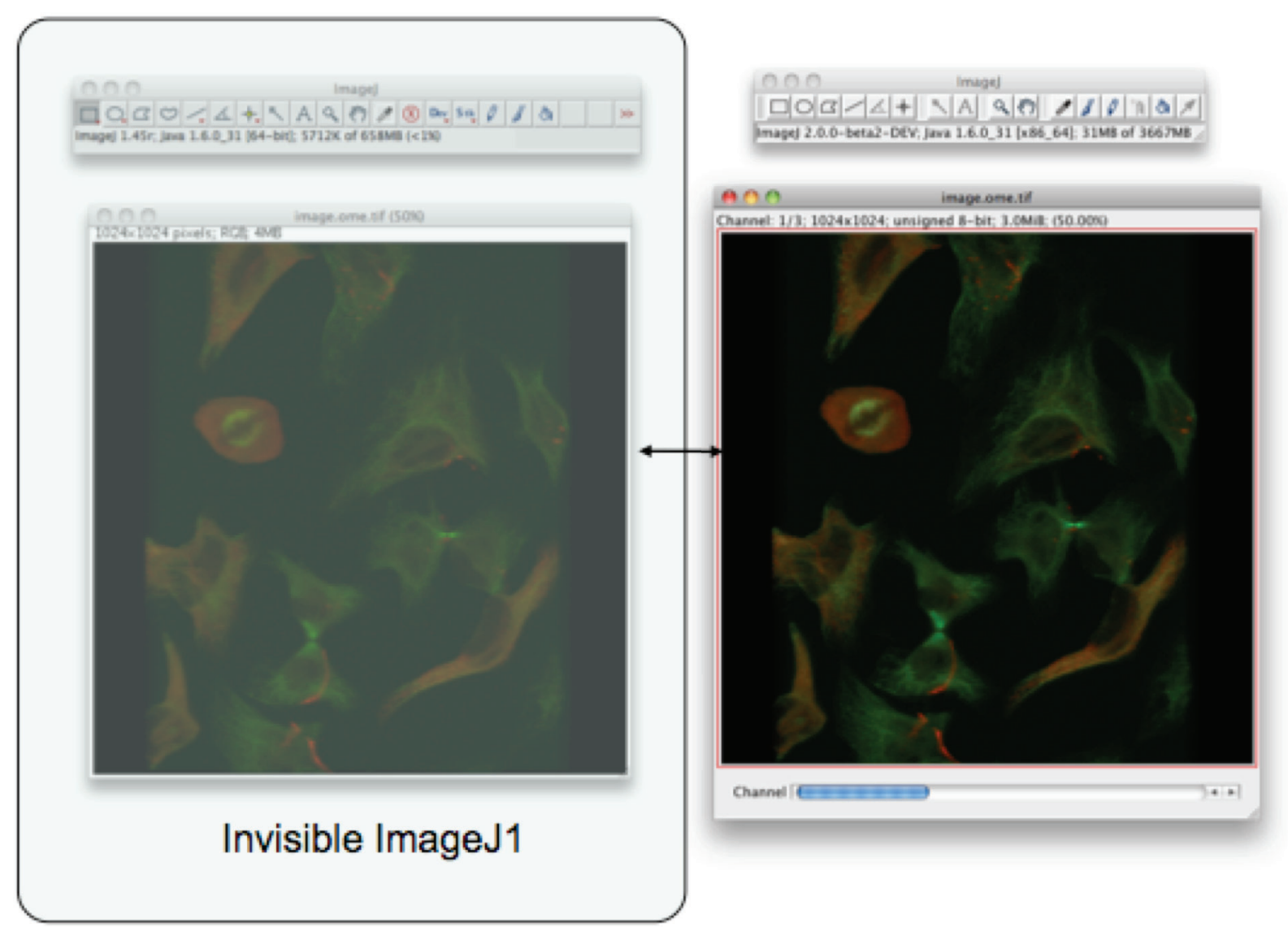

Figure 1: ImageJ2 provides backwards compatibility with ImageJ1 via an integrated "legacy layer" which translates between "legacy" and "modern" data structures. 